\title{
EVALUACIÓN DE LA ACCIÓN SÍSMICA PARA TUNJA - COLOMBIA
}

\author{
SEISMIC INPUT ASSESSMENT FOR TUNJA - COLOMBIA
}

Gladys Patricia Molina Molina

Ingeniera Civil, Reconocedora Predial, Unidad Administrativa Especial Catastro, Bogotá D.C., Colombia, gpmolinam@hotmail.com

Andrés José Alfaro Castillo

Ingeniero Civil, MSc, Ingeniero Especialista, Ingetec S.A.,

Profesor Ingeniería Civil Universidad de La Salle, Bogotá D.C., Colombia, alfaro@ciees.org

Fecha de recepción: 27 de abril de 2009

Fecha de aprobación: 11 de agosto de 2009

\section{RESUMEN}

Este artículo presenta tres aspectos de la evaluación de la acción sísmica para la ciudad de Tunja, en primer lugar una evaluación probabilista de la amenaza sísmica, en segundo lugar se presenta un espectro sísmico de diseño en roca para un período de retorno de 475 años y por último un mapa de periodos predominantes del suelo a partir de estudios de Relación espectral HVSR superpuesto a la clasificación de suelos en superficie.

Palabras clave: Tunja, Amenaza, Espectro Sísmico, Periodos Predominantes, Nakamura, HVSR, Colombia.

\begin{abstract}
This paper brings up three aspects of a seismic input assessment for the city of Tunja, first, a probabilistic earthquake hazard assessment, secondly, a rock design earthquake spectrum for a 475-year return. And finally a soil predominant period map using a Horizontal to Vertical Spectral Ratio -HVSR- over imposed to the surface soil classification.
\end{abstract}

Keywords: Tunja, earthquake hazard, earthquake spectrum, predominant periods, Nakamura, HVSR. 


\section{INTRODUCCIÓN}

Tunja está ubicada en la cordillera Oriental colombiana a los $05^{\circ} 32^{\prime} 07^{\prime \prime}$ de latitud Norte y $73^{\circ} 22^{\prime} 04^{\prime \prime}$ de longitud Oeste, se encuentra en un área propensa a la actividad sísmica; en la zona se desarrollan varios sistemas de fallas activas. Según datos históricos, en los últimos 500 años la sismicidad de Tunja y su área cercana ha sido destacada. Las fallas geológicas más activas y de mayor relevancia en el departamento de Boyacá, cuya capital es Tunja, son: Guaicaramo o del Piedemonte llanero, Santa María, Soapaga-Salinas, Río Suárez y Bahamón y Boyacá. La concentración de daños en algunas zonas de la ciudad parece indicar la presencia de suelos blandos que generan aumento de la señal sísmica.

Dentro de los 300 sismos reportados para Tunja hasta julio de 2009 , se destacan en especial cinco que han causado daños a estructuras y que han producido importantes pérdidas: el primero de estos fue el del 12 de julio de 1785 a las 7:45 a.m., que según informes históricos fue el mayor terremoto y el más destructor, que afectó la catedral, la gobernación y el colegio Boyacá (Ramírez, 1975).

Para estudiar la posibilidad de generación de efectos locales, Guacaneme $(2000,2006)$ analizó los daños generados por los sismos de mayor importancia para Tunja, su ubicación y las estructuras que se vieron afectadas.

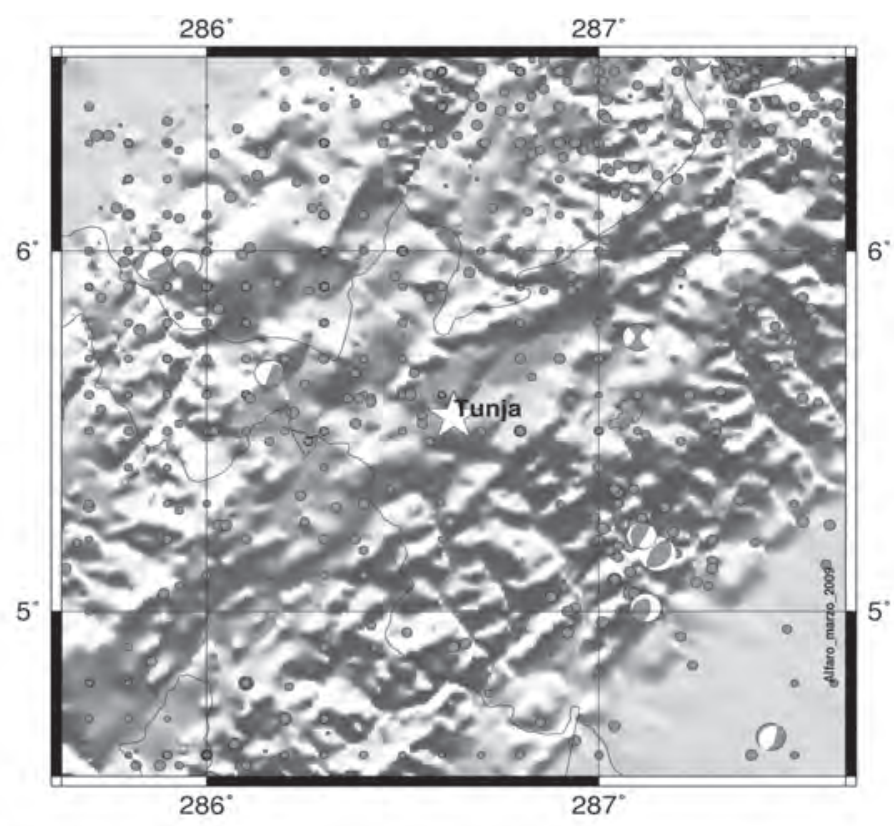

GMT 2009 Mar 24 13:52:36

Figura 1. Epicentros y mecanismos focales en el área de estudio. 
En primer lugar, se observó la sismicidad histórica en un área de 40.000 Km² comprendida entre las coordenadas 4.54 y 6.54 latitud Norte y entre 72.37 y 74.37 longitud Oeste. Después esta área se redujo a $20.445 \mathrm{Km}^{2}$, debido a que no se tienen registros de sismos en toda la zona. La figura 1 muestra los epicentros y los mecanismos focales más importantes de la zona de influencia sísmica para Tunja.

\section{EVALUACIÓN PROBABILÍSTICA DE LA AMENAZA SÍSMICA REGIONAL PARA TUNJA}

La evaluación probabilística de la amenaza sísmica para Tunja se realizó siguiendo a Hanks y Cornell (1994) y a Takada (2005), método que consiste en analizar los sismos que ocurrieron en el área de estudio así como su localización, magnitud y tiempo de ocurrencia. Se analizaron sismos históricos e instrumentales que han sido reportados desde 1566 hasta el año 2009 y para conocer los eventos sísmicos se consultaron los catálogos U.S Geological Survey (USGS, 2009), SISRA ( Programa para la mitigación de los efectos de los terremotos en la región andina) y PDE (Preliminary Determination of Epicenters) disponibles en Internet.

Para el análisis de la evaluación probabilística se usaron dos periodos de tiempo, el primero tomando toda la serie histórica de 443 años desde 1566 a 2009 con una tasa de 0.0905 sismos/año, y el segundo tomando un tiempo parcial en el cual los sismos tuvieran aproximadamente una distribución de tipo Poisson en el tiempo y el espacio para 73 años con una tasa de 0.5069 sismos /año. Sin embargo el método de Hanks y Cornell (1994) asume que la ocurrencia de los sismos en la región de interés se aproxima a un Proceso de Poisson, con una tasa constante de sismos en el tiempo y en el espacio. Por lo tanto solo el periodo de 1935 a 2009 puede ser considerado como un proceso de Poisson.

La evaluación de la amenaza sísmica involucra la estimación del movimiento del suelo que será producido por futuros sismos. Esto se logra normalmente a través del uso de relaciones de atenuación que predicen valores de parámetros seleccionados del movimiento del suelo, en este caso de la aceleración, como una función de otros parámetros sísmicos tales como la magnitud y la distancia de la fuente sísmica al sitio.

Existen muchas ecuaciones para determinar la atenuación del movimiento del suelo. Para este caso se tomaron seis ecuaciones de Douglas $(2001,2002)$ que para su desarrollo utilizan una gran base de datos y una magnitud expresada en Ms. Las ecuaciones utilizadas fueron Patwardhan et al (1978), Fukushima et al. (1988) \& Fukushima \& Tanaka (1990), Sarma y Srbulov $(1996,1998)$, Smit et al (2000) y Ambraseys \& Douglas (2000). Las ecuaciones de atenuación permiten estimar la distancia a la cual se pueden producir aceleraciones dadas para magnitudes dadas. 
Para determinar la curva de amenaza sísmica se procede a combinar la tasa anual de ocurrencia de sismos con una magnitud dada por unidad de área. Finalmente, el total de la tasa media anual de ocurrencia del movimiento sísmico mayor que una aceleración a) dada, es la sumatoria de las contribuciones de las diferentes magnitudes. En la figura 2 se presentan los análisis realizados con datos de 1935 a 2009.

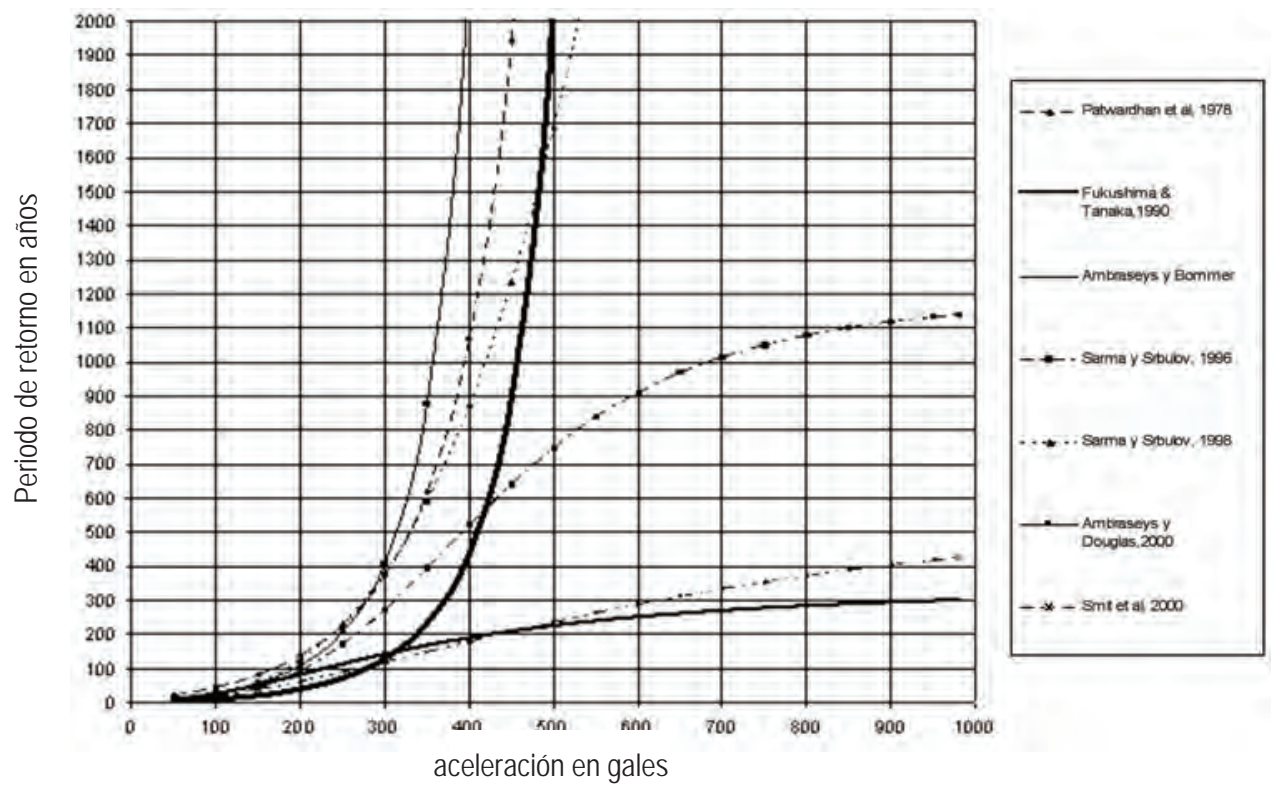

Figura 2. Curvas de Amenaza Sísmica para Tunja 1935- 2009

En el campo de la ingeniería sismológica es común expresar estos resultados en tiempo de retorno. Para labores de diseño y revisión estructural comúnmente se utilizan los periodos de retorno de 475, 1000 y 2000 años, la tabla 1 muestra los resultados de la investigación.

Tabla 1. Valores de aceleración en Gales para 475, 1000 y 2000 años. Datos de 1935-2009

\begin{tabular}{|c|c|c|c|c|c|c|c|}
\hline \multirow[b]{2}{*}{$\begin{array}{l}\text { Periodo } \\
\text { de } \\
\text { Retorno } \\
\text { TR } \\
\text { en años }\end{array}$} & \multicolumn{6}{|c|}{ Aceleración en Gales para Tiempo Parcial (70años) } & \multirow[b]{2}{*}{$\begin{array}{c}\text { Acelera- } \\
\text { ción en } \\
\text { gales } \\
\text { Según } \\
\text { NSR-98 }\end{array}$} \\
\hline & $\begin{array}{l}\text { Atenuación } \\
\text { Patwardhan } \\
\text { et al., (1978) } \\
\text { Idriss(1978) } \\
\end{array}$ & \begin{tabular}{|l|} 
Atenuación \\
Fukushima \\
et al. (1998) \& \\
Fukushima \& \\
Takada (1990)
\end{tabular} & \begin{tabular}{|l} 
Atenuación \\
Sarma y \\
Srbulov \\
$(1996)$ \\
\end{tabular} & \begin{tabular}{|l} 
Atenuación \\
Sarma y \\
Srbulov \\
(1998) \\
\end{tabular} & $\begin{array}{l}\text { Atenuación } \\
\text { Ambraseys } \\
\text { \& Douglas } \\
(2000)\end{array}$ & Promedio & \\
\hline 475 & 325 & 405 & 380 & 325 & 310 & 349 & 196 \\
\hline 1000 & 395 & 460 & - & 420 & 360 & 409 & \\
\hline 2000 & 425 & 500 & - & 530 & 395 & 463 & \\
\hline
\end{tabular}




\section{ESPECTRO SÍSMICO DE DISEÑO PARA LA CIUDAD DE TUNJA}

Consultando el catálogo de NOAA (1996) hay 36 registros horizontales y 14 verticales correspondientes a nueve sismos, que cumplen con las siguientes características: contenidos frecuenciales coherentes con las fuentes sismogénicas colombianas, principalmente del sistema de Guaicáramo; de los estudios de Alfaro-Arias et al. (2001) y Arévalo et al. (2003), quienes analizaron los contenidos frecuenciales de cuatro fuentes sísmicas colombianas: Nido de Bucaramanga, Sistema de Fallas de Atrato, Sistema de Fallas de Romeral y el Sistema de Fallas de Guaicáramo, se ha estimado que los contenidos frecuenciales están entre $0.03 \mathrm{~Hz}$ y $8.9 \mathrm{~Hz}$, distancias epicentrales de acuerdo con el Estudio Geológico Regional; las magnitudes similares a las máximas históricas registradas en la zona de interés y las aceleraciones escalables. Los registros sísmicos corresponden a los eventos de: Long Beach (1933), San Fernando (1971), Sitka (1972), Alaska Subduction (1974), Imperial Valley (1979), Morgan Hill (1984), Valparaíso segunda réplica (1985), Loma Prieta (1989); adicionalmente un registro en roca del sismo del Quindío (1999). Se analizó el registro en Quetame-Colombia del evento del 25 de mayo de 2008 en roca a 9 kilómetros del epicentro, sin embargo, a pesar de las altas aceleraciones pico presentaba un comportamiento que se puede considerar anómalo y por esta razón no se tuvo en cuenta en el resultado.

Los espectros sísmicos de respuesta fueron calculados con el programa SPECEQ/UQ (Nigam y Jennings, 1968). Para la determinación de los espectros sísmicos de diseño se han utilizado métodos estadísticos (Housner, 1941; Newmark y Hall, 1981). Se calculó la media, desviación estándar y una confiabilidad del 90\%.

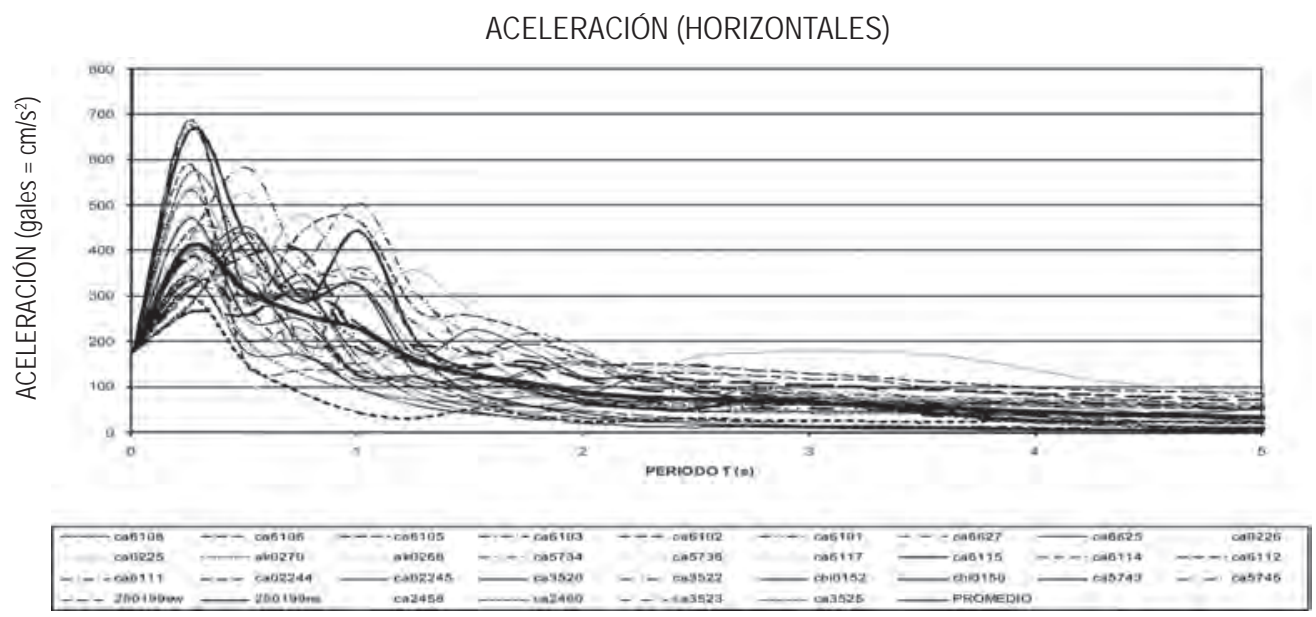

Figura 3. Espectros Sísmicos de Respuesta en Roca

La figura 3 muestra los espectros de respuesta de aceleración en roca de los registros horizontales escalados. En tanto que la figura 4 muestra el espectro de diseño 
propuesto para un período de 0.0 s y una aceleración de 400 gales que crece linealmente hasta 1000 gales para un período de 0.15 segundos, este valor permanece constante hasta un período de 0.454 segundos, a partir de este punto decrece con la siguiente fórmula: $\mathrm{a}=454 T^{-1}$ hasta un período de 5.0 segundos.

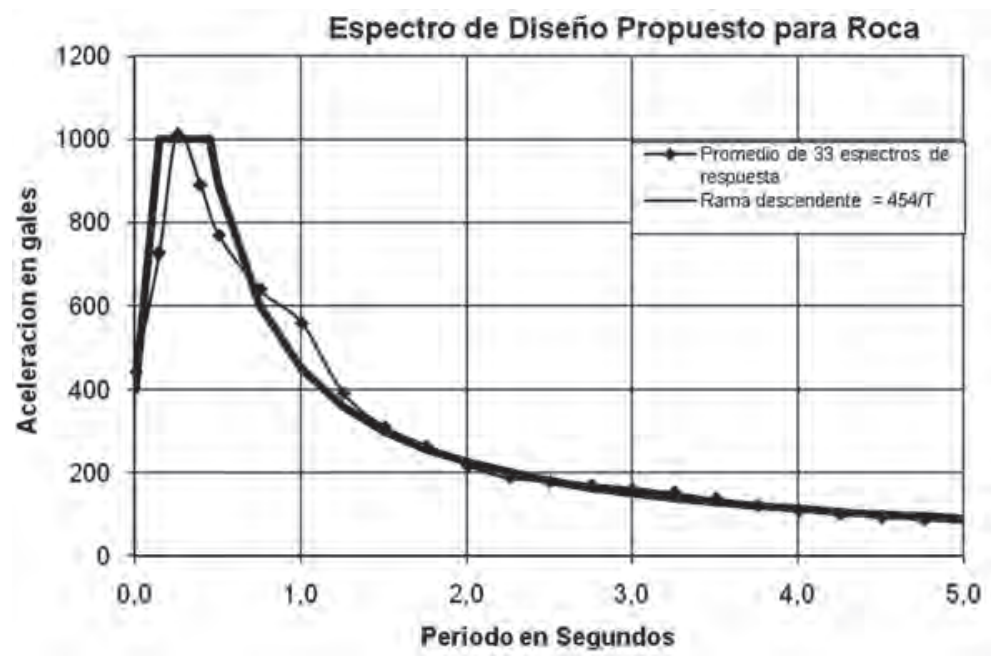

Figura 4. Espectro Sísmico de Diseño en Roca TR = 475 años; la línea delgada corresponde al promedio y la línea gruesa es la idealización del espectro (Molina, 2008).

La tabla 2 presenta los valores del espectro sísmico de diseño en roca.

Tabla 2. Espectro sísmico de diseño en roca $T_{R}=475$ años.

\begin{tabular}{|c|c|c|}
\hline $\mathbf{T}$ (seg) & Aa (gales) & $\% \mathrm{~g}$ \\
\hline 0.00 & 400 & 40.8 \\
\hline 0.15 & 1000 & 101.9 \\
\hline 0.454 & 1000 & 101.9 \\
\hline $0.72-5.00$ & $454 \mathrm{~T}-1$ & variable \\
\hline
\end{tabular}

\section{MAPA DE PERIODOS PREDOMINANTES DEL SUELO A PARTIR DE ESTUDIOS DE RELACIÓN ESPECTRAL HVSR}

Para la elaboración del mapa Periodos dominantes de los suelos de la ciudad de Tunja se tomaron como referencia datos de periodos predominantes del suelo, de Páez y Rincón (2001). La utilización de microtemblores para caracterizar zonas se realiza 
desde los años 50, la metodología propuesta por Nakamura en 1989 para la estimación de los períodos predominantes de amplificación da resultados fiables, de forma rápida y a bajo costo. Se presenta un mapa con los períodos predominantes de los suelos de la ciudad de Tunja estimados a partir de la medición de microtemblores (Figura 5). El análisis de la información ha permitido diferenciar tipos de suelo, de acuerdo con características geotécnicas. Para los sitios localizados sobre afloramientos rocosos se aprecia un período predominante de $0.06 \mathrm{~s}$.

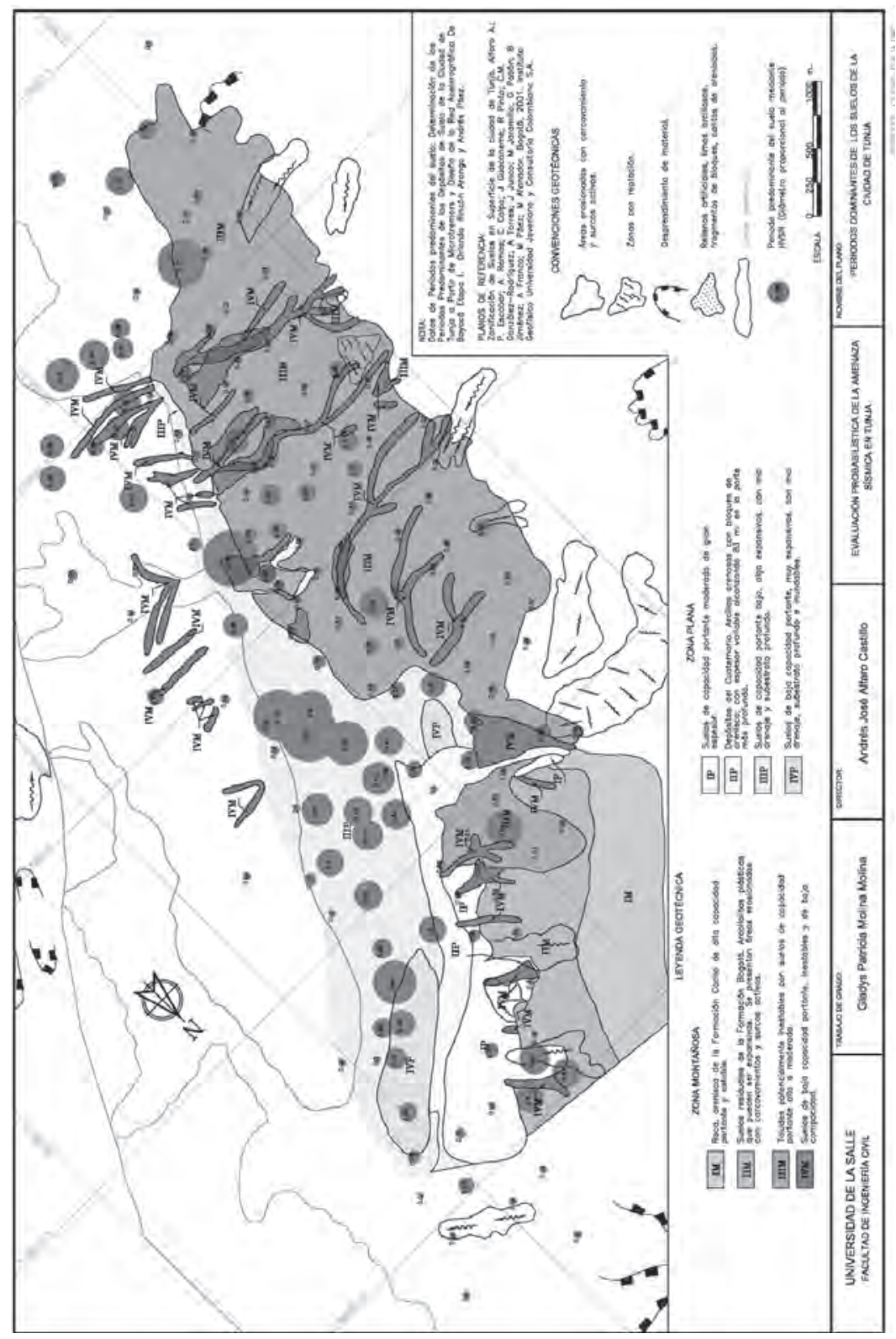

Figura 5. Periodos Predominantes de los suelos de la ciudad de Tunja (Molina, 2008). 
El método de Nakamura (1989) para la estimación de los períodos predominantes del suelo determina a partir de registros en superficie, la función de transferencia de las capas de suelo desde el basamento rocoso a la superficie, mediante la relación entre el espectro de Fourier de la componente horizontal y el de la componente vertical. Las mediciones se realizaron con un equipo triaxial de Kinemetrics, con respuesta plana hasta $50 \mathrm{~Hz}$.

Del mapa se puede observar que los periodos predominantes del suelo de Tunja se encuentran concentrados la mayoría en los suelos de tipo IIIP, IIIM e IVM. De los cuales las profundidades mayores se encuentran en el tipo IIIP que son suelos de capacidad portante baja, algo expansivos, con mal drenaje y subestrato profundo ya que en este suelo se ven reflejados los periodos de mayor duración. Los tipos de suelo IIM y IVM que cuentan con taludes altamente inestables muestran los diferentes tipos de periodos y es donde se ve la mayor concentración. Aunque a profundidades menores los otros tipos de suelo presentan periodos predominantes pero no con tanta incidencia.

\section{CONCLUSIONES}

La ciudad de Tunja está situada en una zona de amenaza sísmica importante, con evidencia de sismos que han causado daños y víctimas.

Para el análisis de la evaluación probabilística se usaron dos periodos de tiempo, uno tomando toda la serie histórica de 1566 a 2009 de 443 años con una tasa de 0.0905 sismos/año, y el otro tomando un tiempo parcial en el cual los sismos tuvieran aproximadamente una distribución de tipo Poisson en el tiempo y el espacio para 73 años con una tasa de 0.5069 sismos /año. Los análisis mostraron aceleraciones pico más altas para el periodo de tiempo corto, es decir, que puede ser más confiable este periodo por sustentarse en datos que fueron tomados durante una época en la cual es más fidedigno el registro en cuanto a localización y magnitud.

Las aceleraciones pico para un $\Delta T$ de 73 años van de 310 a 405 gales para un periodo de retorno de 475 años, 360 a 460 gales para un periodo de retorno de 1000 años y 395 a 530 gales para un periodo de retorno de 2000 años.

El valor de las aceleraciones pico promedio en roca para un tipo parcial de 73 años y periodos de retorno de 475, 1000 y 2000 años respectivamente fueron 346, 460 y 468 gales. De acuerdo a la norma sismo-resistente la aceleración pico para Tunja es de 196 gales. Se encontró un valor promedio de 346 Gales para un periodo de retorno de 475 años, lo cual difiere en un $76.5 \%$ del valor propuesto por la Norma.

El espectro de diseño en roca propuesto: para un período de 0.0 s se obtuvo una aceleración de 400 gales que crece linealmente hasta 1000 gales para un período de 0.15 
segundos, este valor permanece constante hasta un período de 0.454 segundos, a partir de este punto decrece con la siguiente fórmula: $\mathrm{a}=454 T^{-1}$ hasta un período de 5.0 segundos.

Finalmente se presenta un mapa de periodos predominantes del suelo a partir de estudios de relación espectral HVSR, útil para posteriores estudios de amenaza sísmica local. Dentro de los trabajos de microzonificación sísmica de núcleos urbanos, la determinación de las amplificaciones de la señal sísmica y la banda de frecuencias asociadas es una labor prioritaria.

El método de Nakamura, por su sencillez tanto en la parte experimental, como en el procesamiento de la información, se ha convertido en una buena alternativa para identificar los períodos predominantes del suelo. En el caso de la experimentación de Tunja, la aplicación del método ha permitido diferenciar zonas.

Sería deseable ampliar los estudios realizando investigaciones de campo de las fallas en la zona de interés, para tener una aproximación determinista del nivel de amenaza.

\section{AGRADECIMIENTOS}

El programa para el procesamiento HVSR fue realizado por Laura Chavarria (1997) del Servei Geològic de Catalunya - Institut Cartogràfic de Catalunya. Los datos de Harvard corresponden al proyecto CMT (Dziewonski et al., 1981; Dziewonski y Woodhouse, 1983; Woodhouse y Dziewonski, 1984). La Figura 1 fue desarrollada utilizando GMT (Wessel y Smith, 2004).

\section{REFERENCIAS}

[1] Alfaro-Arias C., Van Hissenhoven R. y ALFARO A. (2001) Caracterización en el Dominio de la Frecuencia de Sismos Colombianos. Memorias XIV Jornadas Estructurales de la Ingeniería de Colombia, Bogotá.

[2] Alfaro, A. (2006) Caracterización Dinámica de Suelos Mediante Microtemblores - HVSR y Arreglos. Universidad de La Salle. ISBN 978-958-9290-73-6. 103 págs.

[3] Ambraseys, N., \& Douglas, J. 2000 (Aug). Reappraisal of the effect of vertical ground motions on response. ESEE Report 00-4. Department of Civil and Environmental Engineering, Imperial College, London.

[4] Arévalo, N. T. Caicedo, R. Van Hissenhoven y Alfaro A. (2003) Contenidos Frecuenciales de sismos colombianos registrados en BOCO (Bogotá) y SDV (Santo 
Domingo, Venezuela) (1994-1996) Ingeniería y Universidad. Vol 7, No 1, pp 1931.

[5] Chavarria, L., 1997. Manuals de programes per a l'anàlisi freqüencial i temporal d'enregistraments sísmics digitats. Informe SGC No. GS93/97.

[6] Douglas, J. A. Comprehensive Worldwide Summary of Strong-Motion Attenuation Relationships for Peak Ground Acceleration and Spectral Ordinates (1969 to 2000). London: Imperial College of Science, Technology and Medicine, 2001.

[7] Douglas, J.A. Errata and additions to ESEE Report No. 01-1 "A comprehensive worldwide for peak ground acceleration and spectral ordinates (1969to 2000)". London: Imperial College of Sciencie Technology and Medicine, 2002.

[8] Dziewonski, A. y Woodhouse, J. An experiment in the systematic study of global seismicity: centroid-moment tensor solutions for 201 moderate and large earthquakes of 1981, J. Geophys. Res., 88 (1983): 3247-3271.

[9] Dziewonski, A., Chou, T. y Woodhouse, J. «Determination of earthquake source parameters from waveform data for studies of global and regional seismicity». J. Geophys. Res., 86 (1981): 2825-2852.

[10] Fukushima, Y., Tanaka, T., \& Kataoka, S. 1988. A new attenuation relationship for peak ground acceleration derived from strong-motion accelerograms. Pages 343348 of: Proceedings of Ninth World Conference on Earthquake Engineering, vol. II.

[11] Fukushima, Y., \& Tanaka, T. 1990. A new attenuation relation for peak horizontal acceleration of strong earthquake ground motion in Japan. Bulletin of the Seismological Society of America, 80(4), 757-783.

[12] Guacaneme, J. Microzonificación Sísmica Preliminar de Tunja. Trabajo de Grado. Pontificia Universidad Javeriana. Bogotá, 2000.

[13] Guacaneme, J. Zonificación de suelos en superficie de la ciudad de Tunja, Colombia. Revista Epsilon 6, pp 29-44. 2006.

[14] Hanks, T. y Cornell, C. Probabilistic Seismic Hazard Analysis: A Beginner's Guide. In Proceedings of the Fifth Symposium on Current Issues Related to Nuclear Power Plant Structures, Equipment and Piping, North Carolina State University, Raleigh. (1994): I/1-1 to I/1-17.

[15] Harvard (2009) <http://www.seismology.harvard.edu/projects/CMT/> 
[16] Housner, G.W. (1941) Calculations of the response of an oscillator to arbitrary ground motion, Bull. Seism. Soc. Am. 31, 143-149.

[17] Molina Molina, P., Evaluación Probabilística de la Amenaza Sísmica regional para Tunja. Trabajo de Grado. Ingeniería Civil. Universidad de La Salle. 2008.

[18] Nakamura, Y., 1989. A method for dynamic characteristics estimation of surface using microtremor on the ground surface, Quarterly Rep of RTRF, 30, 1, February, 25-33.

[19] Newmark, N.M. y Hall, W.J. (1982) Earthquake Spectra and design. Monograph, Earthquake Engineering Research Institute, Berkeley, California.

[20] Nigam, N.C. and Jennings, P.C. (1969) Calculation of Response Spectra From Strong Motion Earthquake Records, Bull. Seis. Soc. Amer., 59:2, pp. 909-922.

[21] Páez, C. y O. Rincón, Determinación de los periodos predominantes de los depósitos de suelo de la ciudad de Tunja a partir de microtremors y diseño de la red acelerográfica de Boyacá etapa I: Trabajo de Grado Facultad de Ingeniería Universidad Pedagógica y Tecnológica de Colombia.

[22] Patwardhan, K.; Sadigh, I. M.; Idriss and Youngs, R. 1978. Attenuation of Strong Motion Effect of Site Conditions. Transmission Path Characteristics and Focal Depths, submitted to the Bull. Seismological Society of America.

[23] Ramírez J.E. (1975) Historia de los terremotos en Colombia. Instituto Geográfico Agustín Codazzi-IGAC. Bogotá. 285 pág.

[24] Sarma, S.K., \& Srbulov, M. 1996. A simplified method for prediction of kinematic soil-foundation interaction effects on peak horizontal acceleration of a rigid foundation. Earthquake Engineering and Structural Dynamics, 25(8), 815-836.

[25] Sarma, S.K., \& Srbulov, M. 1998. A uniform estimation of some basic ground motion parameters. Journal of Earthquake Engineering, 2(2), 267-287.

[26] Smit, P.; Arzoumanian, V.; Javakhishvili, Z.; Arefiev, S.; Mayer-Rosa, D.; Balassanian, S., \& Chelidze, T.; 2000. The digital accelerograph network in the Caucasus. In: Balassanian, S. (ed), Earthquake Hazard and Seismic Risk Reduction - Advances in Natural and Technological Hazards Research. Kluwer Academic Publishers. Presented at 2nd International Conference on Earthquake Hazard and Seismic Risk Reduction, Yerevan, Armenia, 15/9/1998-21/9/1998. 
[27] Takada, T. "Seismic Macro Zonation". International Institute of Seismology and Earthquake Engineering. Lectures Notes. 2005.

[28] USGS - United States Geological Survey. URL: <http://neic.usgs.gov/neis/epic/ epic_rect.html> [2009].

[29] Wessel, P. y Smith, W. The Generic Mapping Tools Version 4 - Technical Reference and Cookbook. http://gmt.soest.hawaii.edu [2004].

[30] Woodhouse, J. y Dziewonski, A. «Mapping the upper mantle: three dimensional modelling of Earth structure by inversion of seismic waveforms». J. Geophys. Res. 89 (1984): 5953-5986. 